Diabetologe 2021 · 17:678-679

https://doi.org/10.1007/s11428-021-00802-3

Online publiziert: 23. Juli 2021

(c) Springer Medizin Verlag $\mathrm{GmbH}$, ein Teil von Springer Nature 2021

\section{Erratum zu: Diagnostik, Therapie und Verlaufskontrolle des Diabetes mellitus im Kindes- und Jugendalter}

Andreas Neu' ${ }^{1}$ Jutta Bürger-Büsing ${ }^{2} \cdot$ Thomas Danne $^{3} \cdot$ Axel Dost $^{4} \cdot$ Martin Holder $^{5}$. Reinhard W. Holl ${ }^{6}$. Paul-Martin Holterhus ${ }^{7} \cdot$ Thomas Kapellen $^{8}$. Beate Karges ${ }^{9}$. Olga Kordonouri ${ }^{3} \cdot$ Karin Lange $^{10}$. Susanne Müller ${ }^{11} \cdot$ Klemens Raile $^{12}$. Roland Schweizer ${ }^{1}$. Simone von Sengbusch ${ }^{13} \cdot$ Rainer Stachow $^{14} \cdot$ Verena Wagner $^{15}$. Susanna Wiegand ${ }^{12} \cdot$ Ralph Ziegler $^{16}$

'Klinik für Kinder- und Jugendmedizin, Universitätsklinikum Tübingen, Tübingen, Deutschland

${ }^{2}$ Diabeteszentrum, Bund diabetischer Kinder und Jugendlicher e. V., Kaiserslautern, Deutschland

${ }^{3}$ Kinder- und Jugendkrankenhaus AUF DER BULT, Hannover, Deutschland

${ }^{4}$ Klinik für Kinder- und Jugendmedizin, Universitätsklinikum Jena, Jena, Deutschland

${ }^{5}$ Olgahospital, Klinikum Stuttgart, Stuttgart, Deutschland

${ }^{6}$ Institut für Epidemiologie und medizinische Biometrie, ZIBMT, Universität Ulm, Ulm, Deutschland

${ }^{7}$ Klinik für Allgemeine Pädiatrie, Universitätsklinikum Schleswig-Holstein, Campus Kiel, Kiel, Deutschland

${ }^{8}$ Klinik und Poliklinik für Kinder- und Jugendmedizin, Universitätsklinikum Leipzig, Leipzig, Deutschland

${ }^{9}$ Sektion Endokrinologie und Diabetologie, Universitätsklinikum, RWTH Aachen, Aachen, Deutschland

${ }^{10}$ Medizinische Psychologie, Medizinische Hochschule Hannover, Hannover, Deutschland

"Praxis für Ernährungsberatung, Ennepetal, Deutschland

${ }^{12}$ Virchow-Klinikum, Charité, Universitätsmedizin Berlin, Berlin, Deutschland

${ }^{13}$ Klinik für Kinder- und Jugendmedizin, Universitätsklinikum Schleswig-Holstein, Campus Lübeck, Lübeck, Deutschland

${ }^{14}$ Fachklinik Sylt für Kinder und Jugendliche, Westerland, Deutschland

${ }^{15}$ Gemeinschaftspraxis für Kinder- und Jugendmedizin, Rostock, Deutschland

${ }^{16}$ Praxis für Kinder- und Jugendmedizin, Diabetologische Schwerpunktpraxis, Münster, Deutschland

\section{Erratum zu:}

Diabetologe 2021

https://doi.org/10.1007/s11428-021-

00769-1

Im Beitrag wurden im Literaturverzeichnis unter den Nummern 27., 53., 78., 105., 128., 153., 177., 202., 227., 254., 279., 306., 331., 359., 382., 404., 430., 458., 482., 509., 533., 560., 585., 610., 636. und 649. die jeweiligen Literaturquellen fehlerhaft dargestellt. Wir bitten diese Fehler zu entschuldigen und die korrekten Literaturangaben zu berücksichtigen.

Der vollständige und korrigierte Artikel steht Ihnen auf www.springermedizin.de zur Verfügung. Bitte geben Sie dort den Beitragstitel in die Suche ein.

\section{Korrespondenzadresse}

Prof. Dr. med. Andreas Neu

Klinik für Kinder- und Jugendmedizin, Universitätsklinikum Tübingen Hoppe-Seyler-Straße 1, 72076 Tübingen, Deutschland andreas.neu@med.uni-tuebingen.de

\section{PD Dr. med. Thomas Kapellen} Klinik und Poliklinik für Kinder- und Jugendmedizin, Universitätsklinikum Leipzig Liebigstraße 20 22, 04103 Leipzig, Deutschland thomas.kapellen@medizin.uni-leipzig.de 
Hier steht eine Anzeige.

黑 Springer 